\title{
ESTADO DE CONSERVACIÓN DE Fitzroya cupressoides EN LA REGIÓN DE LOS LAGOS, CHILE. DESAFÍOS PARA SU CONSERVACIÓN Y RESTAURACIÓN
}

\author{
Bannister, Jan R. ${ }^{1}$; Urrutia-Jalabert, Rocío \\ Travieso, Germán ${ }^{1}$ y Galindo, Nicole
}

\section{RESUMEN}

Los alerzales de la región de Los Lagos han sufrido una larga historia de más de cuatro siglos de explotación indiscriminada. Actualmente estos bosques cubren solo un $42,9 \%$ de su superficie original, y su especie dominante, el alerce, está actualmente clasificada como en peligro de extinción.

En el presente estudio, en base a coberturas regionales de información geográfica del Catastro de Recursos Vegetacionales de Chile (CONAF), se caracteriza la situación actual de los alerzales de la región de Los Lagos, se describe la matriz que los circunda, y se discute sobre los principales desafíos para su conservación y restauración.

Los resultados indican que del total de los alerzales ubicados en la región de Los Lagos (208.251 ha), 81,2 \% (169.112 ha) se encuentran en la cordillera de los Andes, 12,6\% (26.189 ha) en la cordillera del Sarao, $6 \%$ (12.567 ha) en la cordillera de la Costa de Chiloé y $0,2 \%$ (384,2 ha) en la depresión intermedia.

Los alerzales de todos los sectores presentan un alto grado de fragmentación, con una baja proporción del paisaje cubierta por estos, bajos índices de diversidad y cohesión de parches, y altos índices de división del paisaje.

En este escenario, resaltan los alerzales de la cordillera de la costa de Osorno y Llanquihue (Sarao) como los más fragmentados con 1,5 parches cada 100 ha, la menor superficie promedio de parche (66,4 ha), y un $67,9 \%$ de su superficie en estado de renoval, lo que indica que además de estar severamente fragmentados, estos bosques denotan un alto grado de alteración. Además, en este sector no existen áreas protegidas del Servicio Nacional de Áreas Silvestres Protegidas (SNASPE), por lo que no hay protección alguna para la especie.

Caso aparte, lo representan los pequeños y escasos ocho fragmentos de alerce que aún existen en la depresión intermedia (0,84 a 260,9 ha), los cuales están enfrentados a una gran presión antrópica $(66,1 \%$ de la matriz circundante son praderas y matorrales), y por esto, están en peligro crítico de desaparecer.

En este contexto, urge aumentar la protección de los alerzales de la región de Los Lagos, especialmente de aquellos ubicados en la depresión intermedia y la Cordillera de la Costa de las provincias de Osorno y Llanquihue. Además, se debe fomentar la ejecución de iniciativas de

\footnotetext{
${ }^{1}$ Investigador, Instituto Forestal, Oficina Chiloé, Chile. jbannister@infor.cl

${ }^{2}$ Investigadora Instituto Forestal, Sede Los Ríos, Chile

Instituto de Conservación, Biodiversidad y Territorio, Facultad de Ciencia Forestales y Recursos Naturales, Universidad Austral de Chile.

Centro de Ciencia del Clima y la Resiliencia, CR2, Santiago, Chile.
} 
restauración de alerzales a nivel regional, tratando de aumentar el tamaño de los fragmentos, mejorando la conectividad entre estos y aumentando la viabilidad de que sean habitados por alerce.

Por último, en las próximas décadas la región de los Lagos sufrirá grandes cambios en su clima, por lo cual se requiere mayor investigación para analizar el efecto de estos cambios en los alerzales y así poder planificar una estrategia de conservación y restauración de largo plazo que asegure la sobrevivencia de la especie.

Palabras clave: Bosques adultos, estructura de bosques, fragmentación, coníferas longevas, Chile

\section{SUMMARY}

The Fitzroya cupressoides dominated forests (alerzales) of Los Lagos region have suffered a long history of more than four centuries of indiscriminate exploitation. Currently these forests only cover $42.9 \%$ of their original area, and their dominant species, alerce, is at present classified as endangered. In this study, the current situation of the alerzales in Los Lagos region is characterized, the surrounding matrix of these forests is described, and the main challenges for their conservation and restoration are discussed. For this, in this work the regional geographic information coverage based on the Chilean Vegetation Resources Cadastre (CONAF) is used.

The results indicate that from the 208,251 ha of alerzales in Los Lagos region, $81.2 \%$ $(169,112 \mathrm{ha})$ are in the Andes Mountains, $12.6 \%$ (26,189 ha) in Sarao, 6\% (12,567 ha) in Chiloé and $0.2 \%$ (384.2 ha) in the central depression. Alerzales of all sectors present a high degree of fragmentation, with a low proportion of the landscape covered by them, low indices of patch diversity and patch cohesion, and high indices of landscape division. In this scenario, the alerzales of the coastal range of Osorno and Llanquihue (Sarao) stand out as the most fragmented with 1.5 patches per $100 \mathrm{ha}$, the smallest average patch area (66.4 ha), and $67.9 \%$ of their area as second growth forests, which indicates that they are not only severely fragmented, but also present high degradation. Furthermore, there are no public protected areas in this zone, so there is no protection at all for the species. A case apart, are the small and scarce eight alerce fragments that still exist in the central depression ( 0.84 to $260.9 \mathrm{ha}$ ), which are facing a great anthropogenic pressure $(66.1 \%$ of the surrounding matrix are grasslands and shrubs), and because of this, they are in critical danger of disappearing.

In this context, it is urgent to increase the protection of alerzales in Los Lagos region, especially those alerzales located in the central depression and the coastal cordillera of the Osorno and Llanquihue provinces. In addition, the implementation of restoration initiatives in these forests should be encouraged at the regional level, trying to increase the size of the fragments, improving the connectivity between them and increasing the viability of them of being inhabited by alerce. Finally, Los Lagos region will undergo major changes in its climate in the coming decades, so more research is required to analyze the effect of these changes on alerce forests and thus be able to plan a regional-scale and long-term conservation and restoration strategy for these forests.

Keywords: Chile, forest structure, fragmentation, long-lived conifers, old-growth forests 


\section{INTRODUCCIÓN}

Una de las especies más impresionantes e icónicas del hemisferio sur debido a su importancia científica y cultural es Fitzroya cupressoides (Molina) I.M.Johnst. comúnmente llamado alerce (Wolodarsky-Franke y Lara, 2005). Esta conífera forma parte de los bosques templados lluviosos del sur de Sudamérica, es una de las especies más longevas del mundo y forma bosques densos, pudiendo llegar a vivir más de 3.600 años (Lara y Villalba, 1993; Urrutia-Jalabert et al., 2015a). Esta especie endémica del sur de Chile y Argentina tiene una distribución disyunta entre los $39^{\circ} 50^{\prime}$ y los $42^{\circ} 30^{\prime} \mathrm{S}$ en la cordillera de la Costa, depresión intermedia y en la cordillera de los Andes de Chile y áreas adyacentes de Argentina (Donoso, 1993). A pesar de la distribución acotada de estos bosques, su importancia ha sido relevada a nivel internacional principalmente por dos aspectos: su gran longevidad y su capacidad de almacenar grandes cantidades de carbono (Lara y Villalba, 1993; Urrutia-Jalabert et al., 2015a).

Debido a su longevidad, alerce tiene una importancia científica trascendental como archivo ambiental, permitiendo registrar las condiciones climáticas de siglos, e incluso milenios. Es así como a través de sus anillos de crecimiento se pudo reconstruir la temperatura máxima del sur de Sudamérica para los últimos 5.680 años, la reconstrucción climática más larga del hemisferio sur (Lara et al., 2020). Por otra parte, si bien los bosques de alerce están dentro de los bosques lluviosos de más baja productividad en el mundo por su lento crecimiento, estos son capaces de acumular grandes cantidades de carbono, encontrándose entre los bosques más masivos en biomasa del planeta (Urrutia-Jalabert et al., 2015a). Es así como estos bosques antiguos pueden actuar como sumideros de carbono en el largo plazo, debido a sus bajas tasas de mortalidad y a la durabilidad extraordinaria de su madera, la que puede permanecer en el bosque por milenios (Urrutia-Jalabert et al., 2015a). En cuanto al crecimiento anual en diámetro de la especie, este es bastante bajo, con incrementos que fluctúan entre 0,6 y $6 \mathrm{~mm}$, dependiendo de la edad y las condiciones de sitio (Lara et al., 2003).

Debido a la belleza y durabilidad de su madera, alerce ha sufrido una larga historia de más de cuatro siglos de explotación (Wolodarsky-Franke y Lara, 2005). Su aprovechamiento indiscriminado llevó a que actualmente estos bosques cubran solo un $42,9 \%$ de su superficie original hacia 1550 (Lara et al., 1999; Wolodarsky-Franke y Lara, 2003) y que estén actualmente clasificados como en peligro de extinción (UICN, 2020). Medidas de protección de la especie incluyen su incorporación el año 1973 al Apéndice I de la Convención CITES (Convención sobre el Comercio Internacional de Especies Amenazadas de Fauna y Flora Silvestres), prohibiéndose su comercio internacional, y a partir del año 1976 su declaración como Monumento Natural, lo cual prohíbe su corta en Chile (Wodolarsky y Lara, 2003). Actualmente, los bosques del tipo forestal alerce cubren una superficie de 216.130 ha a nivel nacional (CONAF, 2020), con estimaciones al año 2007 que ascienden a un volumen total de 25,7 millones de $\mathrm{m}^{3}$ de alerce vivo, 2,9 millones de $\mathrm{m}^{3}$ de alerce muerto en pie y 5,9 millones de $\mathrm{m}^{3}$ de residuos de alerce en el suelo (Bahamondez, 2007).

Dentro de la distribución de alerce, una situación crítica de conservación la constituyen los bosques que crecen en la depresión intermedia, donde solo quedan escasos parches de pequeña superficie (Lara et al., 2008). Estos bosques se encuentran por tanto altamente fragmentados y amenazados por pastoreo, falta de regeneración, incendios y la expansión urbana desmedida, especialmente durante los últimos años (Fraver et al., 1999; Silla et al., 2002; UrrutiaJalabert et al., 2018a). Pese a su actual protección en Chile, aún ocurren cortas ilegales de alerce, especialmente en la cordillera de la Costa, y es altamente amenazado por incendios que son causados casi exclusivamente por el ser humano (Wolodarsky-Franke y Lara, 2005; CONAF, 2020). Los incendios ocurridos durante la colonización europea en el siglo XIX son los que mayormente explican su casi desaparición de la depresión intermedia entre Puerto Varas y Puerto 
Montt (Veblen et al., 1976). No obstante, incendios ocurridos durante el verano seco y caluroso de 1997-1998 destruyeron más de 9.400 ha de estos bosques en el sector de Fresia en la Cordillera de la Costa (Lara et al., 2003). Dadas las condiciones más secas y calurosas asociadas al cambio climático en el centro-sur de Chile, es esperable que los incendios se hagan más recurrentes y que afecten una mayor superficie (Urrutia-Jalabert et al., 2018b), lo cual constituye una gran amenaza para los bosques de la especie. Por último, si bien hasta el momento se ha encontrado que alerce puede ser relativamente resistente a las condiciones climáticas proyectadas para la zona (UrrutiaJalabert et al., 2018a; Urrutia-Jalabert et al., 2020), existen variaciones dentro de la especie, por lo que condiciones más secas y calurosas pueden significar un riesgo para su sobrevivencia en algunos sitios (Urrutia-Jalabert et al., 2015b, Urrutia-Jalabert et al., 2018a).

Pese a la importancia de estos bosques, existen escasos trabajos publicados en que se muestren experiencias de restauración con la especie. Una de las experiencias mejor estudiadas, es el ensayo establecido en 1998 con fines de conservación genética en la depresión intermedia en las cercanías de Puerto Montt (Fundo Chaqueihua) (Lara et al., 2008). Este ensayo constituye la primera experiencia de restauración ecológica con la especie en el país y en él se evaluó la supervivencia y crecimiento de alerce bajo distintas condiciones de sitio, además de la inducción de conos para la producción de semillas (Lara et al., 2008). Los resultados de este estudio constituyen información valiosa para la selección de sitios y aplicación de tratamientos, encontrándose un porcentaje superior al $75 \%$ de supervivencia al cabo de cinco años, al utilizarse plantas de semilla sin fertilización.

\section{OBJETIVOS}

Dentro del contexto que el $96,3 \%$ de los bosques de alerce se concentran en la región de Los Lagos (Bahamondez, 2007; CONAF 2020), el presente estudio tiene por objetivo:

a) Caracterizar la situación actual de los alerzales en la región de Los Lagos, en términos de estructura, composición y métricas de fragmentación.

b) Describir la matriz circundante a los alerzales de la región de Los Lagos, en términos de uso del suelo y estructura de bosques.

c) Discutir los principales desafíos para la conservación y restauración de estos bosques, bajo el contexto del cambio climático y presiones antrópicas crecientes en la región. Esta información será de gran importancia para planificar en el mediano plazo una estrategia para la conservación y restauración de estos bosques en la región de Los Lagos.

\section{MATERIAL Y MÉTODO}

\section{Área de Estudio}

El área de estudio comprende los límites administrativos de la región de Los Lagos en el sur de Chile. La región comprende una superficie total de 4.840 .836 ha, de las cuales el 58,4\% está conformado por bosque nativo (CONAF, 2020). Esta región se encuentra dentro de las regiones biogeográficas templadas norte y sur de Chile, incluyendo flora de ambas regiones (Bannister et al., 2012). El clima regional es templado oceánico con influencia mediterránea en la zona norte (Di Castri y Hajek, 1976). La fisiografía de la región está caracterizada por la cordillera de los Andes al este, la cordillera de la Costa al oeste, la depresión intermedia en su parte norte y los archipiélagos al sur. Debido a sus características topográficas, la región presenta un amplio 
gradiente de sitios, siendo la cordillera de los Andes la formación geológica más elevada con un máximo de $3.228 \mathrm{~m}$, seguido de las cordilleras de la Costa continental y de Chiloé, con 1.106 y 840 $\mathrm{m}$, respectivamente.

Para abarcar de buena forma la gran diversidad de sitios en que crecen los alerzales en la región, se definieron cuatro sectores de estudio delimitados por sus características geológicas y edafoclimáticas (Figura $\mathrm{N}^{\circ} 1$ ): Sarao (cordillera de la Costa continental), Chiloé (cordillera de la Costa en el archipiélago de Chiloé), depresión intermedia, y cordillera de los Andes. Debido a su distribución discreta, el sector depresión intermedia está compuesto por solo ocho conglomerados. Cada uno de estos sectores presenta un amplio gradiente altitudinal (Figura $\mathrm{N}^{\circ} 2$ ).

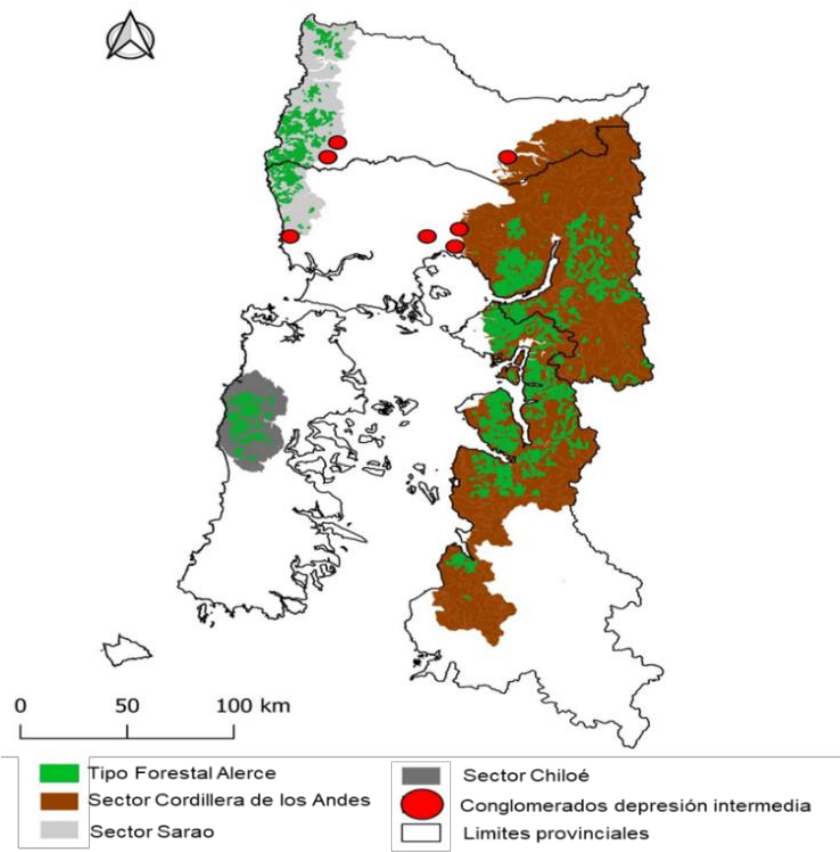

Líneas negras representan límites administrativos regionales y provinciales.

En el caso de la depresión Intermedia se observan solo siete conglomerados ya que dos de ellos se encuentran a $1 \mathrm{~km}$ de distancia.

\section{Figura $\mathrm{N}^{\circ} 1$ \\ DISTRIBUCIÓN DE LOS BOSQUES DEL TIPO FORESTAL ALERCE Y SECTORES DE ESTUDIO EN LA REGIÓN DE LOS LAGOS}

Cada sector incluye todos los bosques de alerce según el Catastro de Recursos Vegetacionales de Chile actualizado al año 2013 para la región de Los Lagos (CONAF, 2020). Sin embargo, para realizar análisis a escala de paisaje, hubo que delimitar detalladamente cada uno de los sectores. Para esto se definieron los límites de los sectores a través de dos criterios, uno geológico basándose en la distribución de la composición de las rocas basales de acuerdo al 
Servicio Nacional de Geología y Minería (SERNAGEOMIN, 2003), y el otro de existencias de acuerdo al Catastro de Recursos Vegetacionales de Chile actualizado al año 2013 (CONAF, 2020).

En primer lugar, según el criterio geológico, se consideró para la cordillera de la Costa, tanto en Chiloé como Sarao, principalmente rocas metamórficas. Para la cordillera de los Andes se consideró principalmente rocas intrusivas y secuencias volcánicas, con una mínima parte también compuesta por rocas metamórficas, y para la depresión intermedia se consideraron principalmente secuencias sedimentarias (SERNAGEOMIN, 2003). Usando este criterio se pudo hacer una predefinición de los límites de cada sector de estudio. En segundo lugar, se generaron cuencas hidrográficas para cada sector de estudio predefinido a través del software SAGA GIS 2.3.2, con el modelo de elevación digital ALOS PALSAR DEM de 12,5 m de resolución espacial, generado por el satélite ALOS de la Agencia Japonesa de Exploración Aeroespacial (JAXA).

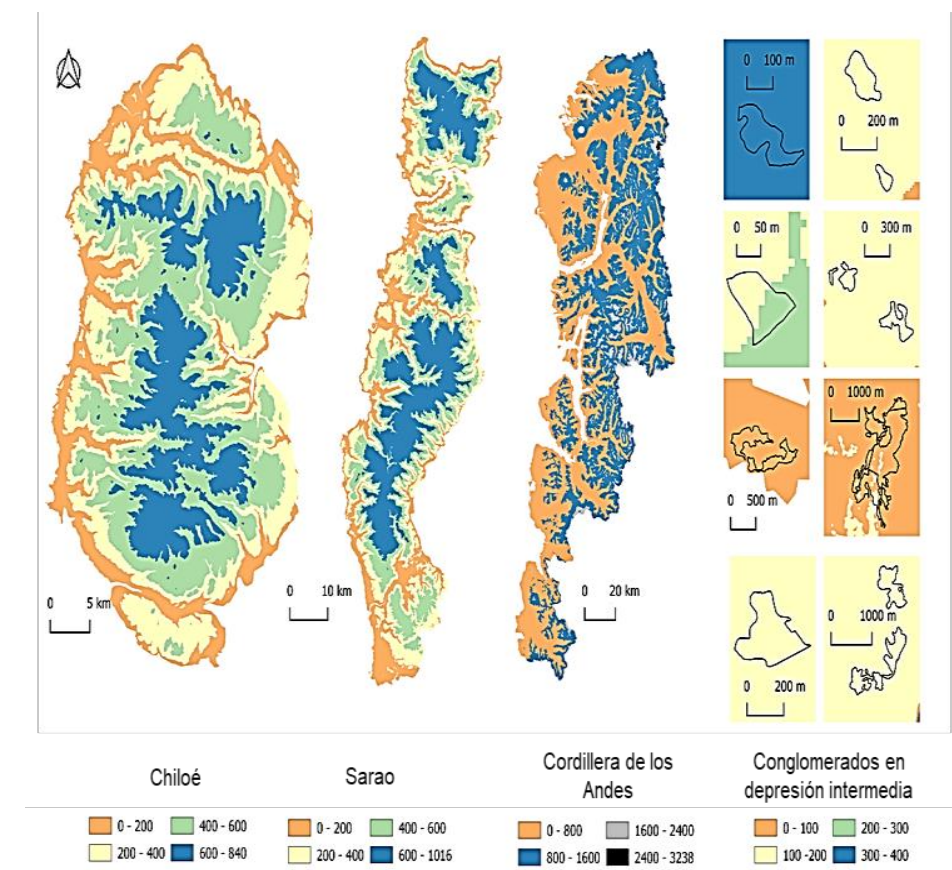

Distintos colores representan distintos rangos altitudinales en cada sector de estudio (msnm)

Figura $\mathrm{N}^{\circ} 2$

RANGOS ALTITUDINALES PARA LOS CUATRO SECTORES DE ESTUDIO

De esta forma, se seleccionaron en cada sector con el criterio geológico aquellas cuencas hidrográficas con cobertura de bosques de alerce, de tal manera que los límites quedaran finalmente definidos por el límite exterior de las cuencas hidrográficas que se ubicaban en los sectores de estudio. En el caso de la cordillera de Los Andes, esta no contaba con una continuidad 
de cuencas con presencia de alerce a lo largo de todo su territorio, por eso excepcionalmente, para evitar mayores subdivisiones, se seleccionaron algunas cuencas sin coberturas de bosques de alerce para conectar el territorio con la siguiente cuenca con existencias de alerce, y así lograr tener un solo sector. Finalmente, para el caso de la depresión intermedia, fueron analizados los bosques de alerce a nivel de conglomerado. Se entiende en este estudio por conglomerado a aquel conjunto de bosques compuestos por uno o más parches de alerce distanciados a no más de $1 \mathrm{~km}$.

Es importante mencionar que, en el caso de la cordillera de la Costa de Chiloé, se eliminaron todos aquellos polígonos definidos como bosques de alerce localizados fuera de la cordillera de Piuchué, debido a que aquellos bosques no corresponden a alerzales, sino a otro tipo de bosques como cipresales (conocimiento práctico de los autores). Para describir la pendiente topográfica en los bosques de alerce, se utilizó el mismo modelo de elevación digital y su interpretación está sujeta a los criterios de CIREN en documento de pautas para estudio de suelos. Para analizar la superficie y distribución de los bosques de alerce se utilizó la cobertura SIG del Catastro de Recursos Vegetacionales de Chile de CONAF, actualizado al año 2013 (CONAF, 2020).

\section{Caracterización de la Estructura, Composición y Distribución de los Bosques de Alerce}

Se consideró como bosques de alerce aquellos polígonos con cobertura del tipo forestal alerce, que según el reglamento técnico del Decreto de Ley 701 , se define como aquella agrupación arbórea o arbustiva en que existe a lo menos un individuo de esta especie por hectárea. Para caracterizar la estructura y composición de los bosques de alerce, se utilizaron cuatro variables presentes en la tabla de atributos del catastro: cobertura, altura, estructura y composición (Cuadro $\mathrm{N}^{\circ} 1$ ). Usando la variable composición se calculó la frecuencia relativa de especies arbóreas y arbustivas en todos los polígonos con presencia de alerce.

\section{VARIABLES DEL CATASTRO UTILIZADAS PARA CARACTERIZAR LOS BOSQUES DE ALERCE}

\begin{tabular}{|l|c|}
\hline Variable & Categorías \\
\hline \multirow{3}{*}{ Cobertura (\%) } & Denso $>75)$ \\
& Semidenso $(50-75)$ \\
& Abierto $(25-50)$ \\
\hline Altura $(m)$ & $2-4$ \\
& $4-8$ \\
& $8-12$ \\
& $12-20$ \\
& $20-32$ \\
Estructura & $>32$ \\
& Bosque adulto \\
& Bosque adulto-renoval \\
& Bosque renoval \\
Composición & Bosque achaparrado \\
\hline
\end{tabular}


La frecuencia relativa de cada especie se estimó calculando el número de polígonos con presencia de cada especie dominante respecto al total de polígonos para cada sector de estudio, existiendo 1.016 polígonos en la cordillera de Los Andes, 1.067 polígonos en Sarao, 297 polígonos en Chiloé y 29 polígonos en la depresión intermedia.

Para este cálculo, no se hizo distinción entre dominancia de especies (rankings 1 a 6 de composición) (Cuadro $\mathrm{N}^{\circ} 1$ ). Además, para analizar la similitud florística entre sectores de estudio se realizó un análisis de clúster usando la presencia de especies arbóreas en cada sector.

El análisis de clúster se basó en el índice de Jaccard y el algoritmo UPGMA (unweighted pair-group average) con soporte estadístico bootstrap con 1000 iteraciones para los diferentes nodos (Bannister et al., 2012; Bannister y Donoso, 2013).

UPGMA es un algoritmo aglomerativo consistente, en donde disimilitudes entre grupos son calculadas como el promedio de todos los pares posibles de miembros de cada grupo (Kreft y Jetz, 2010).

Este enfoque podría estar influenciado por diferentes esfuerzos de muestreo, acceso en los sectores de estudio y las limitaciones del catastro de CONAF, pero debido a que se usaron solamente especies arbóreas para el análisis no debería haber cambios en la tendencia.

\section{Indicadores de Fragmentación de Bosques de Alerce}

Para caracterizar la fragmentación de los bosques de alerce en cada uno de los sectores de estudio, se seleccionaron seis índices de parche, seis índices de paisaje y dos índices de diversidad (Cuadro $\mathrm{N}^{\circ} 2$ ).

Para el cálculo de indicadores de fragmentación en cada uno de los sectores de estudio, se realizó una rasterización del formato gráfico vectorial de los datos espaciales.

La rasterización se realizó a través del software GRASS GIS 7.8.2 utilizando vectores de información binaria, esto es; "bosques de alerce" (valor 1) y "otros usos" (valor 2), para así poder crear rasters de salida con solo dos tipos de información, eliminando de esta manera el factor "polígono" que compone los usos de suelo según CONAF (2020).

Esto fue realizado utilizando una resolución para cada píxel de $20 \times 20 \mathrm{~m}$. Los polígonos pertenecientes a la depresión intermedia estaban constituidos por ocho conglomerados discretos y aislados, por este motivo no se les aplicó análisis de fragmentación.

De esta forma, se realizaron estos análisis solo para los sectores de estudio cordillera de los Andes, Chiloé y Sarao.

Las métricas de fragmentación de bosques fueron calculadas a través el software QGIS Madeira 3.4.15 con el plugin LecoS, diseñado por Jung (2013) para la automatización de análisis relacionados a la ecología del paisaje, basándose en las herramientas desarrolladas por el proyecto opensource FRAGSTATS, y que admite solo archivos raster como input. 
Cuadro $\mathrm{N}^{\circ} 2$

LISTA DE MÉTRICAS DE TIPO PARCHE, PAISAJE Y DIVERSIDAD CALCULADO A TRAVÉS DE LECOS

\begin{tabular}{|c|c|c|}
\hline Métrica & Tipo & Descripción Acotada \\
\hline Paisaje cubierto por alerce (ha) & Paisaje & Superficie cubierta por alerce \\
\hline Proporción de paisaje (\%) & Paisaje & $\begin{array}{l}\text { Proporción de paisaje cubierto por alerce } \\
\text { respecto a la superficie del sector de estudio. }\end{array}$ \\
\hline Número de parches $(n)$ & Parche & $\begin{array}{l}\text { Número total de fragmentos con presencia de } \\
\text { alerce por sector }\end{array}$ \\
\hline Superficie parche más grande (ha) & Parche & - \\
\hline Superficie parche más pequeño (ha) & Parche & - \\
\hline Superficie media de parche (ha) & Parche & $\begin{array}{l}\text { Media aritmética de los fragmentos con } \\
\text { presencia de alerce }\end{array}$ \\
\hline Densidad de parche (n/100ha) & Paisaje & $\begin{array}{l}\text { Número de fragmentos de alerce / superficie } \\
\text { total del sector por } 100 \text { ha }\end{array}$ \\
\hline Longitud de borde (m) & Parche & $\begin{array}{l}\text { Perímetro total de los fragmentos con } \\
\text { presencia de alerce }\end{array}$ \\
\hline Densidad de borde (m/ha) & Parche & $\begin{array}{l}\text { Perímetro total de los fragmentos con } \\
\text { presencia de alerce / superficie total }\end{array}$ \\
\hline Índice del parche más grande & Paisaje & $\begin{array}{l}\text { Importancia del parche más grande (\%). } \\
\text { Cuando se acerca a } 0 \text { el parche más grande } \\
\text { es cada vez más pequeño. } 100 \% \text { : cuando } \\
\text { todo el paisaje consiste en un solo parche }\end{array}$ \\
\hline Índice de cohesión de parches & Paisaje & $\begin{array}{l}\text { Se aproxima a } 0 \text { a medida que la proporción } \\
\text { del paisaje compuesto por bosques de alerce } \\
\text { disminuye y se vuelve cada vez más } \\
\text { subdividido y menos conectado físicamente. } \\
\text { El valor aumenta a medida que aumenta esta } \\
\text { proporción. }\end{array}$ \\
\hline Índice de división de paisaje & Paisaje & $\begin{array}{l}\text { Probabilidad de que dos lugares elegidos al } \\
\text { azar dentro del paisaje bajo estudio no estén } \\
\text { situados en el mismo fragmento de hábitat } \\
\text { contiguo. Por lo tanto, conforme el hábitat se } \\
\text { vuelve cada vez más subdividido en parches } \\
\text { pequeños, la probabilidad aumenta (cercano a } \\
100 \% \text { ) }\end{array}$ \\
\hline Índice de Shannon & Diversidad & $\begin{array}{l}\text { Diversidad de tamaño de parches. Valores }< \\
2 \text {, baja diversidad; valores }>3 \text {, alta diversidad. } \\
\text { Sensible a riqueza. }\end{array}$ \\
\hline Índice de Simpson & Diversidad & $\begin{array}{l}\text { Diversidad de tamaño de parches. Valores } \\
\text { entre } 0 \text { y } 1 \text {. Cercano a } 0 \text {, alta diversidad, } \\
\text { cercano a } 1 \text { baja diversidad. Sensible a } \\
\text { equidad. }\end{array}$ \\
\hline
\end{tabular}

Las variables no explicadas son de común entendimiento 


\section{Caracterización del Paisaje Circundante a los Bosques de Alerce}

Para caracterizar el paisaje o matriz circundante a los bosques de alerce en cada sector de estudio y poder asignar un uso y subuso de suelo al perímetro de cada polígono, se generó para cada sector un área buffer de $5 \mathrm{~m}$ de ancho en el contorno de todos los polígonos definidos como bosques de alerce. La superficie de uso y subuso resultante por cada categoría, fue recalculada en términos de superficie relativa (\%) para interpretar la información como una equivalencia al perímetro colindante, y de esta forma poder obtener una estimación de la matriz circundante a los bosques de alerce.

\section{RESULTADOS}

\section{Distribución, Estructura y Composición de los Bosques Dominados por Alerce}

La región de Los Lagos está cubierta por 2.827.328 ha de bosque nativo, equivalente al $58,4 \%$ del total de su superficie regional. De esta superficie, un total de 208.251 ha corresponde al tipo forestal alerce, lo que representa el $96,3 \%$ de la superficie total del tipo forestal en Chile. A nivel regional, el tipo forestal alerce se encuentra distribuido en las cuatro provincias que componen la región (Palena: 46,9\%, Llanquihue: 37,9\%, Osorno: 9,1\% y Chiloé: $6 \%$ ). En la cordillera de Los Andes se concentra el 81,2 \% (169.112 ha) de la superficie total de los bosques de alerce regional. Sarao por su parte, concentra el 12,6\% (26.189 ha), Chiloé el $6 \%$ (12.567 ha) y la depresión intermedia el 0,2\% (384,2 ha) de los alerzales de la región. En general el $53,7 \%$ de los alerzales se establecen sobre laderas muy escarpadas $(>45 \%$ de pendiente topográfica), y un $36,7 \%$ sobre laderas moderadamente escarpadas a escarpadas (rango $15-45 \%$ ).

En la cordillera de la costa, tanto en Chiloé como Sarao, los alerzales se concentran en pendientes de sobre un $15 \%$, acentuándose la pendiente en la cordillera de los Andes donde el $60 \%$ de la superficie de alerce se encuentra en laderas escarpadas de montaña (Figura $\mathrm{N}^{\circ}$ 3). Caso contrario son los alerzales de la depresión intermedia que se ubican mayoritariamente en terrenos de pendiente "suave" (plano a fuertemente inclinado $<15 \%$ ).

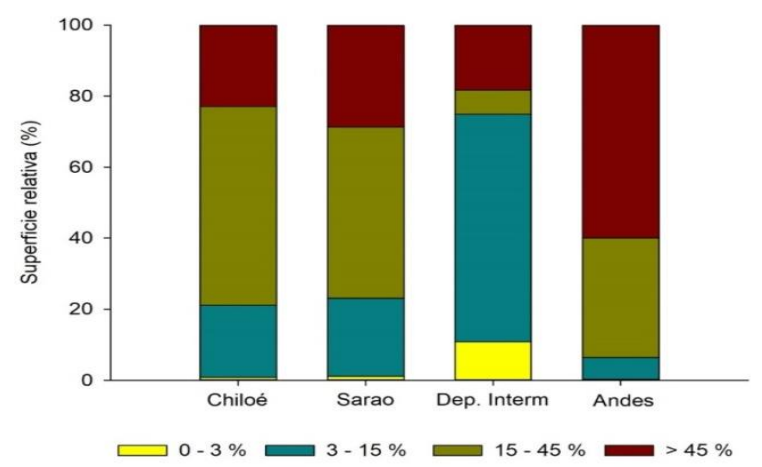

Figura $\mathrm{N}^{\circ} 3$

DISTRIBUCIÓN DE LOS BOSQUES DE ALERCE POR RANGOS DE PENDIENTE PARA CADA SECTOR 
En la cordillera de los Andes y la depresión Intermedia la estructura dominante de los alerzales corresponde a bosque adulto (76,9\%), (Figura $\mathrm{N}^{\circ} 4 \mathrm{a}$ y $4 \mathrm{~b}$ ). En el caso de Sarao y Chiloé, los renovales son la estructura dominante. Los bosques achaparrados tienen su mayor proporción en Chiloé y los de estructura adulto-renoval en Sarao (Figura $\mathrm{N}^{\circ} 4 \mathrm{a}$ y $4 \mathrm{~b}$ ).

En cuanto a la cobertura arbórea, casi la totalidad de los bosques de alerce $(96,1 \%)$ están en las categorías "denso" y "semidenso", es decir, presentan una cobertura de copas mayor a $50 \%$ de su superficie. Los bosques abiertos están representados en mayor medida Chiloé, con un $15,7 \%$ de la superficie total de alerces. En el sector costero, tanto en Chiloé como Sarao, dominan los bosques semidensos en proporciones similares (54,2 \% en promedio para ambos sectores geográficos), y en Los Andes y depresión intermedia es donde dominan los bosques densos (Figura 4c y $4 \mathrm{~d}$ ).

En cuanto a la altura de los bosques de alerce, el rango 12 a $20 \mathrm{~m}$ de altura es el que concentra la mayor superficie ( 82.108 ha, $39,4 \%$ del total de bosques), seguido del rango 8 $12 \mathrm{~m}$ (63.526 ha, 30,5\% del total de bosques). La cordillera de los Andes es el sector con mayor cantidad de alerzales de gran altura con la totalidad de los bosques de sobre $32 \mathrm{~m}$ de altura y sobre el $95 \%$ de los bosques de sobre $20 \mathrm{~m}$ de altura. Por su parte, Chiloé es la zona geográfica con bosques más bajos, ya que su superficie se concentra en un $68,8 \%$ en el rango $4-8 \mathrm{~m}$ (Figura 4e y $4 \mathrm{f})$.

Con respecto a la riqueza de especies arbóreas y arbustivas acompañantes, la depresión intermedia presenta el valor más bajo con 10 especies, seguido por Chiloé (17), Andes (20) y Sarao (23). Los sectores de estudio difieren en cuanto a las especies más frecuentes (Figura $\mathrm{N}^{\circ}$ 5). En Los Andes, Nothofagus betuloides es la especie acompañante más frecuente, con presencia en el $95 \%$ de los polígonos definidos para dicho sector, seguida de varias otras especies con una frecuencia menor al $40 \%$. En Chiloé, son cuatro especies las que presentan frecuencias sobre el $75 \%$, Tepualia stipularis, Drymis winteri, Pilgerodendron uviferum y Nothofagus nítida. Sarao presenta tres especies con frecuencias sobre el $50 \%, D$. winteri, $N$. nítida y $T$. stipularis, y finalmente la depresión intermedia, presenta cinco especies con frecuencias relativas importantes sobre el $55 \%$, siendo las principales $D$. winteri y Luma apiculata con 100 y $68 \%$, respectivamente.

El análisis de clúster para la composición de especies arbóreas mostró un alto coeficiente de correlación cofenético (coef. cof: 0,923) y dos divisiones con alto soporte bootstrap. En primer lugar, un nodo con soporte $100 \%$ que crea dos grupos, uno formado por los bosques de Chiloé y depresión intermedia, y uno formado por los bosques de la cordillera de los Andes y Sarao. En segundo lugar, existe un nodo con soporte de $84 \%$ que separa en dos grupos los bosques de la cordillera de los Andes y Sarao, que presentan si una alta similitud (IJ: 0,619).

Los bosques de Chiloé y la Depresión Intermedia tienen una división con un muy bajo soporte bootstrap de $39 \%$ (Figura $\mathrm{N}^{\circ} 6$ ). Cabe destacar la baja similitud $(\mathrm{IJ}$ : 0,388 ) entre los bosques de alerce de Chiloé y los de Sarao y, entre estos últimos y los ubicados en la depresión intermedia (IJ: 0,333).

Finalmente, los bosques de alerce de los distintos sectores tienen distintos niveles de protección en el Sistema Nacional de Áreas Silvestres Protegidas (SNASPE). Actualmente el $48,6 \%$ de los bosques de los Andes, el $34,8 \%$ de los de Chiloé, el $0 \%$ de los de Sarao, y el $28,7 \%$ de los bosques de la depresión intermedia, se encuentran dentro de un área protegida del SNASPE. A nivel regional el grado de protección asciende a un $41,6 \%$ del total de su superficie. 

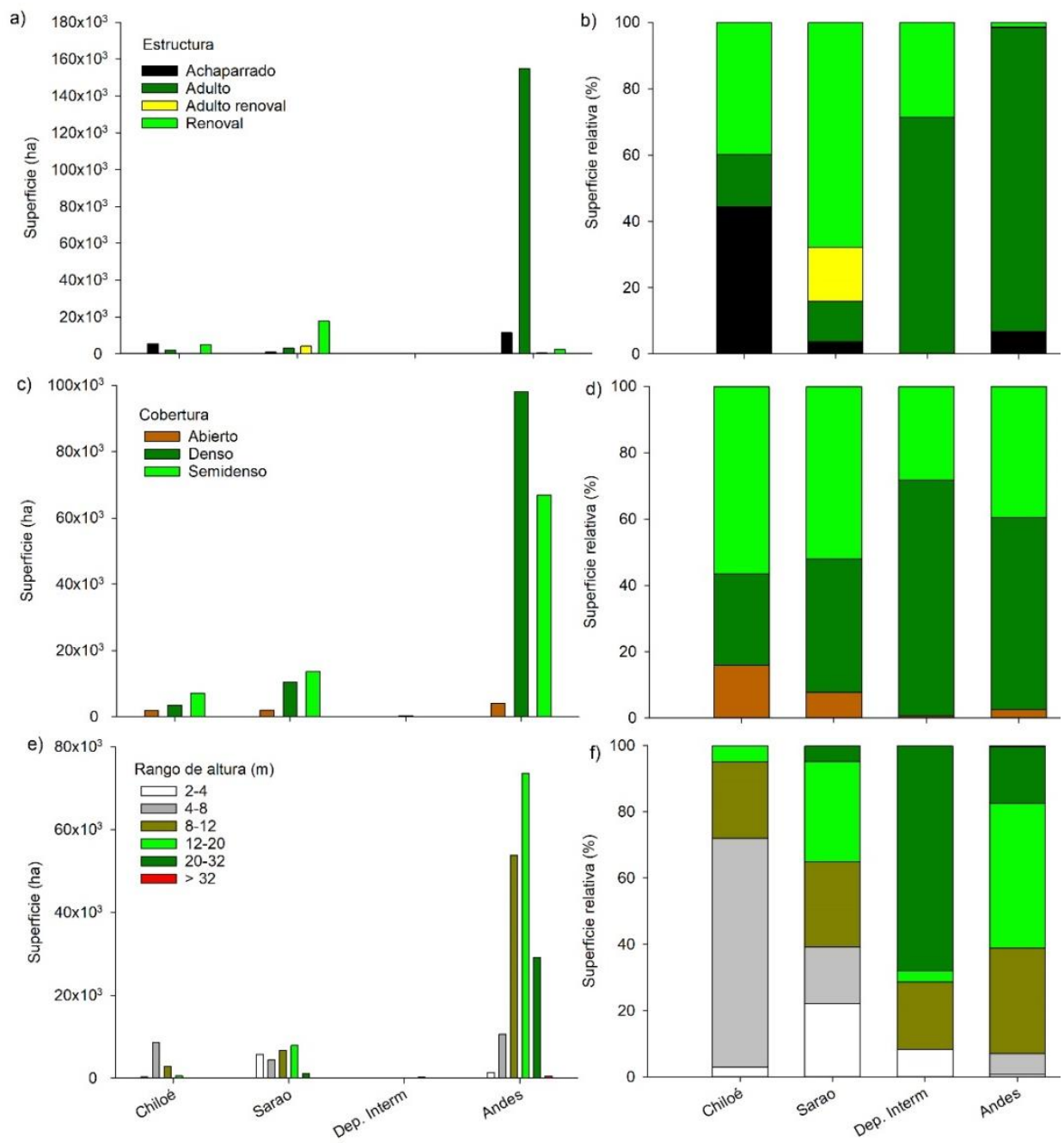

Figura $\mathrm{N}^{\circ} 4$

SUPERFICIE ABSOLUTA Y RELATIVA DE LOS BOSQUES DE ALERCE SEGÚN ESTRUCTURA (a y b), COBERTURA (c y d) Y RANGO DE ALTURAS (e y f), PARA CADA SECTOR DE ESTUDIO EN LA REGIÓN DE LOS LAGOS 

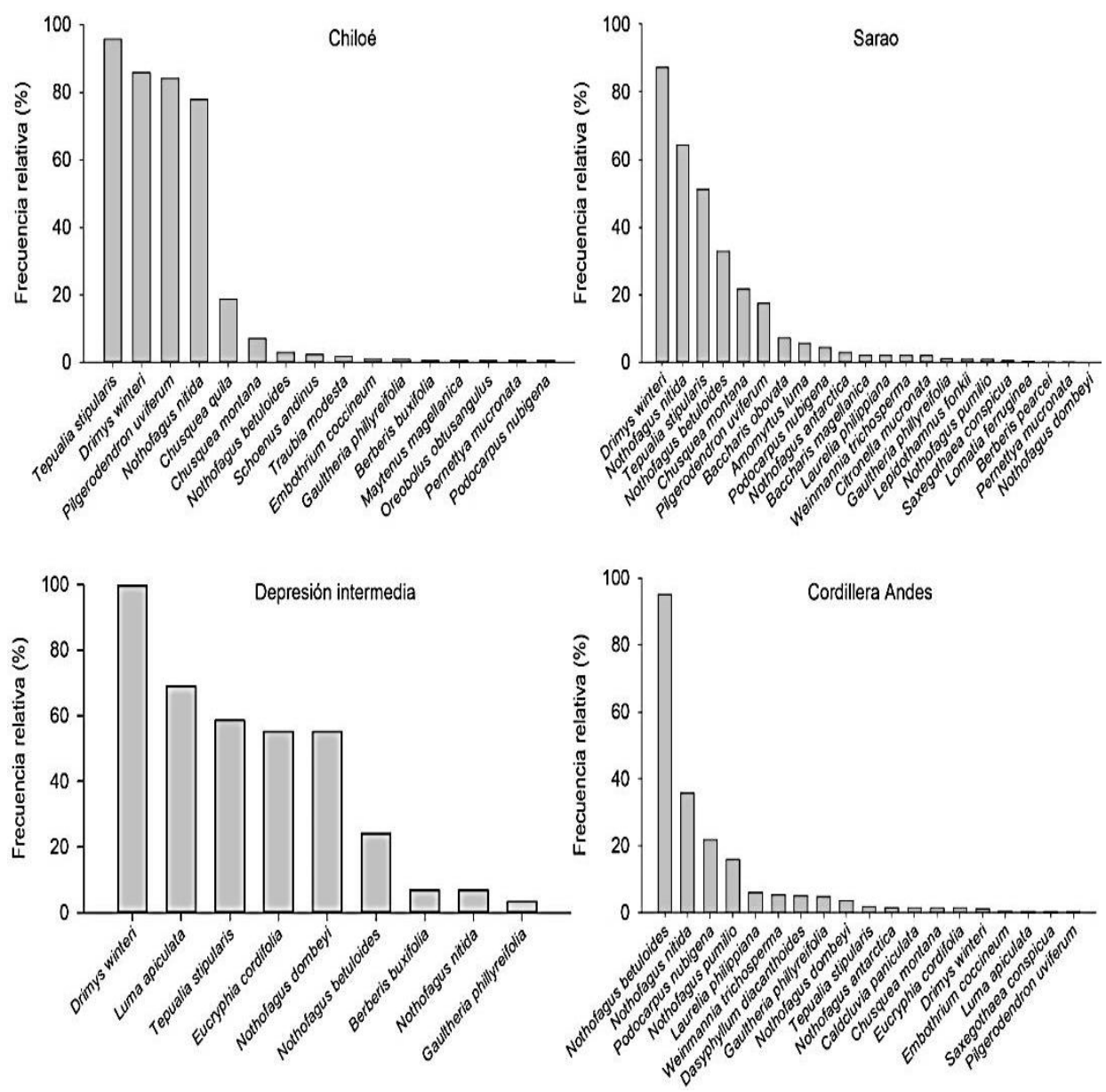

Figura $\mathrm{N}^{\circ} 5$

PORCENTAJE DE OCURRENCIA DE ESPECIES ARBÓREAS Y ARBUSTIVAS EN LOS BOSQUES DOMINADOS POR ALERCE DE LA REGIÓN DE LOS LAGOS 


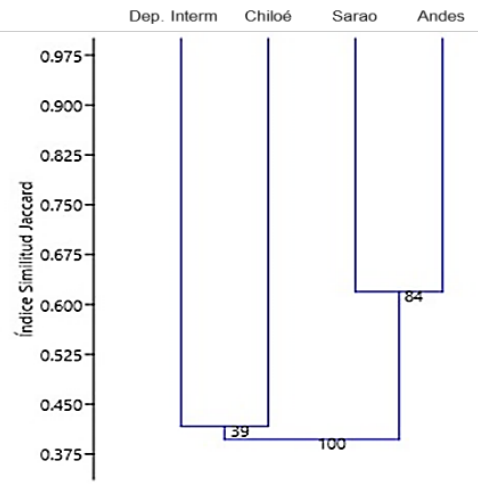

Números cercanos a los nodos representan el soporte bootstrap para cada división.

\section{Figura $\mathrm{N}^{\circ} 6$ \\ ANÁLISIS DE CLÚSTER BASADO EN LA COMPOSICIÓN ARBÓREA DE LOS DISTINTOS SECTORES DE ESTUDIO EN LA REGIÓN DE LOS LAGOS (COEF. COF: 0,923).}

\section{Fragmentación de los Bosques Dominados por Alerce}

Los tres sectores geográficos definidos para el estudio de fragmentación (Sarao, Chiloé y Andes), están cubiertos entre un 7 y $10 \%$ por bosques de alerces (Cuadro $\mathrm{N}^{\circ} 3$ ). En los tres sectores se observa una fragmentación severa, en donde el tamaño de los parches más grandes es muy pequeño en relación al tamaño del paisaje en observación (índice de parche más grande no superó el $2.9 \%)$. Además, el índice de cohesión entre parches fue bajo en todos los sectores $( \pm 10 \%)$ y el índice de división del paisaje fue casi 1 , lo que indica que, si se eligen dentro del paisaje dos puntos al azar, es casi seguro que estos no van a estar ubicados en un mismo fragmento.

Aparte, la gran cantidad de fragmentos en los distintos sectores presentó una baja diversidad de tamaños tanto en los índices de Shannon como Simpson (cercanos a 0). Sin embargo, la superficie de alerzales se encuentra fragmentada de diferente forma en cada sector, siendo Sarao el sector con mayor número de parches (394 parches) de una superficie media de 66,4 ha, cuyo parche de mayor tamaño corresponde a 3.171 ha. Le sigue en número de parches la cordillera de Los Andes con 266 parches, con una superficie media de 636,3 ha y el parche más grande de 17.772 ha, y los bosques de alerce de Chiloé con 124 parches de una superficie media de 101,7 ha y con el parche de mayor superficie de 3.996 ha.

Además, los alerzales de los Andes presentan en relación a los de Sarao y Chiloé, una menor densidad de parche y borde. En este sentido, el mayor grado de fragmentación en Sarao queda también manifestado en una mayor densidad de parche $(1,5$ parches cada 100 ha) respecto a los otros sectores (0,98 en Chiloé y 0,15 en Andes). Eso si la densidad de borde fue similar entre Chiloé y Sarao, lo que indica similar perímetro o contacto con la matriz circundante. Con respecto a la depresión intermedia, este sector cuenta con un muy bajo número de conglomerados (ocho en total), con superficies medias muy variadas que van desde las 0,84 ha hasta las 260,9 ha (Cuadro $\mathrm{N}^{\circ} 4$ ). De los cuales, cuatro conglomerados se encuentran fragmentados en dos o más parches distanciados a no más de $1 \mathrm{~km}$. 


\section{Cuadro $\mathrm{N}^{\circ} 3$ \\ MÉTRICAS DE FRAGMENTACIÓN PARA EL TIPO FORESTAL ALERCE POR SECTOR GEOGRÁFICO. NO INCLUYE DEPRESIÓN INTERMEDIA}

\begin{tabular}{|l|r|r|r|}
\hline Variable & Chiloé & \multicolumn{1}{c|}{ Sarao } & \multicolumn{1}{c|}{ Andes } \\
\hline Paisaje cubierto por alerce (ha) & $12.610,8$ & $26.178,2$ & $169.261,2$ \\
Proporción de paisaje (\%) & 9,16 & 9,78 & 6,99 \\
Número de parches & 124,0 & 394,0 & 266,0 \\
Superficie parche más grande (ha) & $3.996,5$ & $3.171,2$ & $17.772,0$ \\
Superficie parche más pequeño (ha) & 0,04 & 0,04 & 0,04 \\
Superficie media de parche (ha) & 101,7 & 66,4 & 636,3 \\
Densidad de parches (n/100 ha) & 0,98 & 1,50 & 0,15 \\
Longitud de borde (m) & 1.829 .480 & 3.820 .040 & 8.177 .120 \\
Densidad de borde (m/ha) & 145,07 & 145,92 & 48,31 \\
Índice de parche más grande (\%) & 2,90 & 1,18 & 1,13 \\
Índice de cohesión de parche (\%) & 9,93 & 9,91 & 9,99 \\
Índice división de paisaje & 0,998 & 0,999 & 0,999 \\
Índice de Shannon & 0,30 & 0,32 & 0,34 \\
Índice de Simpson & 0,16 & 0,17 & 0,19 \\
\hline
\end{tabular}

* Debido al proceso de rasterización con el que se construyeron estos índices, en esta tabla el paisaje cubierto por alerce por sector (ha) no calza con superficie de alerce basada en polígonos del catastro.

\section{CARACTERÍSTICAS GENERALES DE LOS CONGLOMERADOS DE ALERCE EN LA DEPRESIÓN INTERMEDIA}

\begin{tabular}{|c|c|r|r|l|}
\hline Conglomerado & $\begin{array}{c}\text { Parches } \\
\text { (N }{ }^{\circ}\end{array}$ & $\begin{array}{c}\text { Superficie Media } \\
\text { (ha) }\end{array}$ & $\begin{array}{c}\text { Superficie Total } \\
\text { (ha) }\end{array}$ & \multicolumn{1}{|c|}{ Estructura } \\
\hline 1 & 1 & 1,97 & 1,97 & Renoval \\
2 & 2 & 1,43 & 2,86 & Adulto \\
3 & 1 & 0,84 & 0,84 & Adulto \\
4 & 3 & 3,07 & 9,22 & Adulto \\
5 & 2 & 14,96 & 29,92 & Renoval \\
6 & 1 & 260,9 & 260,9 & Adulto \\
7 & 1 & 6,19 & 6,19 & Renoval \\
8 & 2 & 36,16 & 72,32 & Renoval \\
\hline
\end{tabular}

\section{Paisaje Circundante a los Bosques de Alerce}

La matriz circundante de los bosques de alerce está compuesta principalmente por bosques y praderas-matorrales con el 78,4 y $14,8 \%$ de la matriz, respectivamente (Figura $N^{\circ} 7$ ). La mayor representación de bosques se cumple para los sectores Chiloé, Sarao y Andes, 
siendo más acentuado en estas dos últimas zonas con el 81,4 y $82,4 \%$ de su matriz, respectivamente. En la depresión intermedia esta relación se invierte, y es el $66,1 \%$ de su matriz la que se encuentra con praderas y matorrales. Además, considerando las categorías de los subusos de suelo bosque y praderas-matorrales como un todo, el $84 \%$ de la matriz es bosque nativo.

Los Andes es el sector con más bosque nativo en su matriz circundante $(94,8 \%)$, seguido de Sarao $(81,4 \%)$ y Chiloé $(67,6 \%)$. Los alerzales de la depresión intermedian solo cuentan con un $29,1 \%$ de su matriz con bosque nativo (Figura $\mathrm{N}^{\circ} 7$ ).
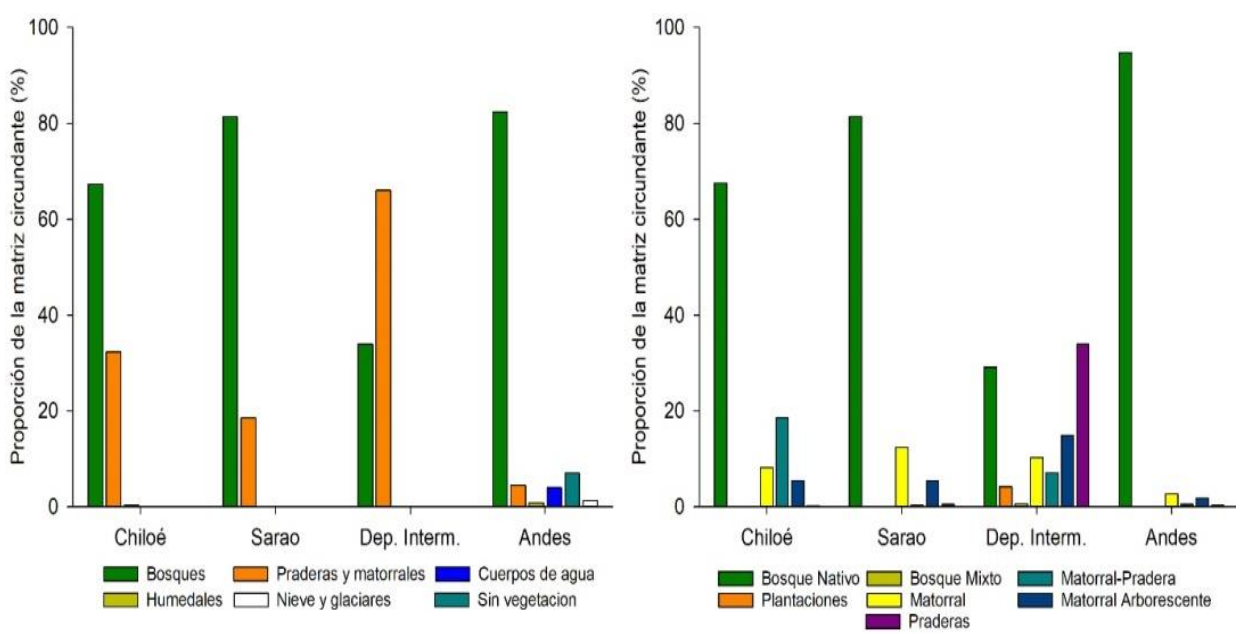

Para el caso de los sub-usos, solo se representan los dos principales usos de suelo; bosques y praderas y matorrales.

Figura $\mathrm{N}^{\circ} 7$

\section{USO Y SUBUSOS EN LA MATRIZ CIRCUNDANTE DE LOS BOSQUES DE ALERCE} POR SECTOR DE ESTUDIO

En cuanto a la estructura de los bosques nativos presentes en la matriz circundante, el $59,3 \%$ son bosques adultos, y el $20,7 \%$ son bosques achaparrados (que podrían estar asociados también a bosques adultos, como es el caso de los tepuales).

Sin embargo, esta estructura de bosque difiere entre los sectores de estudio (Figura $N^{\circ}$ 8). En la depresión intermedia, gran parte de la matriz circundante $(89,8 \%)$ corresponde a renovales. El escenario contrario ocurre en los tres otros sectores, en donde los bosques adultos dominan la matriz circundante (entre un 55 y 69, 5\%). En la cordillera de la costa en tanto (Chiloé y Sarao), existe una proporción relevante de renovales a considerar $( \pm 19-30 \%)$. Los Andes es la zona geográfica que mejores condiciones de matriz circundante presenta, con solo un $9 \%$ de su matriz con renovales. 


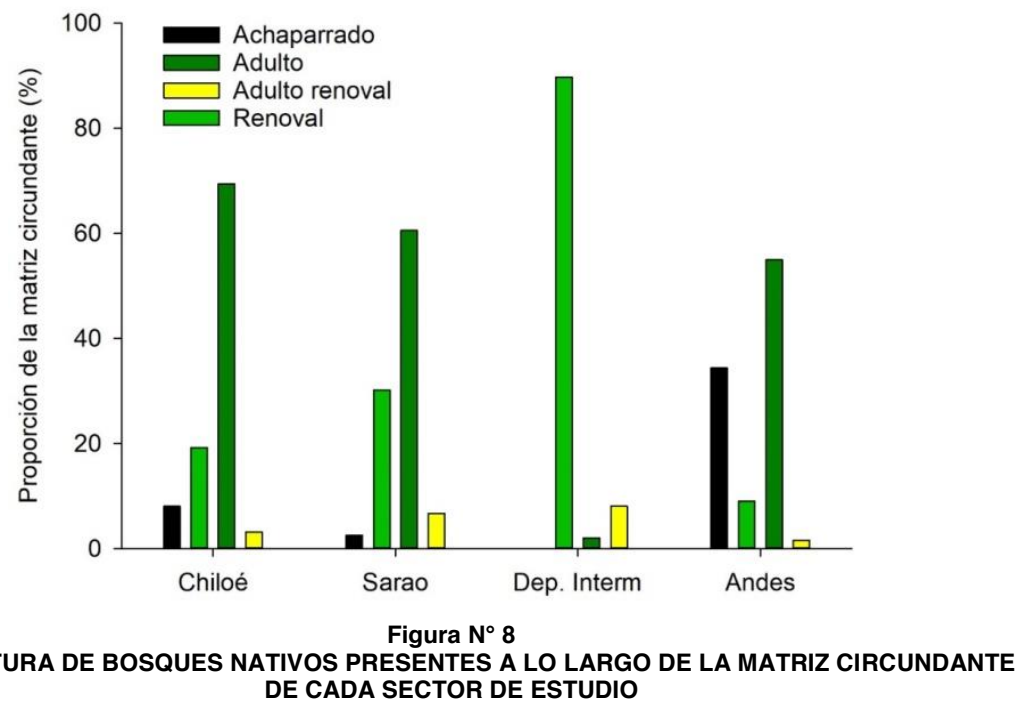

\section{DISCUSIÓN}

\section{Estado de Conservación de los Bosques de Alerce en la Región de Los Lagos}

En la región de Los Lagos se encuentra casi la totalidad de los bosques dominados por alerce en Chile (96,3\%, 208.251 ha), concentrándose estos en la cordillera de los Andes de las provincias de Llanquihue y Palena y en la cordillera de la Costa, principalmente en las provincias de Osorno (cordillera de Sarao) y Chiloé (cordillera de Piuchué). Existen además ocho pequeños fragmentos de alerce en la depresión intermedia, los cuales son remanentes de una gran zona de alerzales que fue quemada durante el siglo XIX (Veblen et al., 1976).

Según el análisis de clúster, los alerzales de la región se pueden dividir a nivel composicional en tres grandes grupos: los Andes, Sarao y Chiloé-Depresión intermedia. Esta división coincide en gran medida con los tres grupos definidos genéticamente por Allnutt et al. (1999), quienes muestran diferencias genéticas entre los alerzales de la cordillera de los Andes, de la Costa norte, y la Costa sur/Depresión Intermedia. Estas diferencias se deberían probablemente al aislamiento genético en el pasado $\mathrm{y}$ al restringido flujo genético en el presente (Allnutt et al., 1999). En este contexto, estudios previos sugieren que alerce estuvo restringido a refugios costeros durante la última glaciación y que las poblaciones de la depresión intermedia corresponderían a relictos glaciales (Allnutt et al., 1999; Villagrán et al., 2004). En este estudio, la unión de los alerzales de Chiloé y la depresión Intermedia como un grupo composicional se debería probablemente a este origen biogeográfico común.

Los cuatro sectores definidos presentan alerzales que se pueden diferenciar en composición arbórea, estructura y cobertura. En general, los bosques de la cordillera de los Andes son bosques adultos de cobertura densa o semidensa, donde $N$. betuloides, $N$. nítida y 
Podocarpus nubigena acompañan a alerce en el dosel dominante. Es así como han sido documentados dos subtipos para los Andes que corresponden a alerce $-N$. nítida y alerce $-N$. betuloides (Donoso et al., 1990). Los bosques de Chiloé son en general achaparrados o renovales de cobertura semidensa a densa donde $T$. stipularis, $D$. winteri, $P$. uviferum y $N$. nítida acompañan a alerce en el dosel dominante. Los bosques de Sarao (la cordillera de la Costa de Osorno y Llanquihue) son en general renovales semidensos a densos, donde $D$. winteri, $N$. nítida, $T$. stipularis y $N$. betuloides dominan el dosel junto a alerce. Estas especies acompañantes son características de los rodales creciendo en la cordillera de la Costa en el continente (Urrutia-Jalabert et al., 2015a).

Por último, en los fragmentos presentes en la depresión intermedia dominan los bosques adultos y densos, donde $D$. winteri, $L$. apiculata, $T$. stipularis y E. cordifolia dominan el dosel junto a alerce. Es importante destacar que, si bien estos bosques aparecen como adultos, la edad que estos rodales alcanzan no es muy avanzada y muchos de ellos corresponden a renovales regenerados después de los incendios del siglo XIX, u otros ocurridos con posterioridad (Silla et al., 2002). El rodal con árboles más viejos y con menos alteración en el área corresponde al Monumento Natural Lahuen Ñadi, donde los árboles más antiguos llegan a los casi 300 años (Silla et al., 2002). De hecho, este rodal es el único que presenta una composición arbórea y de sotobosque significativamente distinta a los demás rodales que han sido estudiados en la depresión intermedia (Fraver et al., 1999).

Los alerzales de la región de Los Lagos presentan un alto grado de fragmentación, con una baja proporción del paisaje cubierta por estos, bajos índices de diversidad de tamaños y cohesión de parches, y altos índices de división del paisaje. Además, los tamaños de los parches más grandes son muy pequeños en relación al tamaño del paisaje. Resaltan entre estos grupos los alerzales de Sarao como los más fragmentados con 1,5 parches cada 100 ha, la superficie promedio de parche menor $(66,4 \mathrm{ha})$, y la superficie menor del parche de mayor tamaño (3.171 ha). Cabe destacar que los bosques de este sector además de estar más fragmentados que los de Chiloé y los Andes, presentan un 67,9\% de su superficie en estado de renoval, lo que indica que además de estar severamente fragmentados, estos bosques presentan también un alto grado de alteración. Esta condición está dada por la ocurrencia histórica de incendios y cortas en esta cordillera (Lara et al., 1999).

Según un inventario de bosques muertos de alerce en las regiones de Los Ríos y Los Lagos, las comunas costeras de la región de Los Lagos concentran altos volúmenes de madera muerta de alerce y una abundante regeneración de la especie (Bahamondez, 2007). Los alerzales de Chiloé por otra parte, presentaron indicadores levemente mejores que Sarao, sin embargo, preocupa que pese a presentar un menor número de fragmentos, los alerzales de Chiloé presentaron una densidad de borde similar, lo que indica que estos fragmentos presentan una exposición similar y no menor que los de Sarao a la matriz circundante.

En el caso de los alerzales de la cordillera de los Andes, estos presentan una menor densidad de parche y borde lo que hace inferir un menor grado de fragmentación dentro de la negativa realidad general que se infiere de los otros indicadores. Caso aparte representan los escasos fragmentos ubicados en la depresión intermedia, que debido a que su fragmentación es tan extrema (solo existen ocho fragmentos en este sector), ni siquiera se pudo realizar los análisis de fragmentación para estos bosques.

En cuanto al estado de protección de alerce en áreas protegidas del SNASPE, pese a no llegar a los niveles de protección que tienen estos bosques en Argentina (85\%, Kitzberger et al., 2000), a nivel general los alerzales de la región de los Lagos presentan una protección de $41,6 \%$. Esto aún resulta insuficiente dado el carácter de especie en peligro de extinción y el 
hecho de que aún existen cortas ilegales que afectan la especie (Wolodarsky-Franke y Lara, 2005, noticias de prensa). Esto cobra importancia, además al considerar que en la cordillera de la Costa de las provincias de Llanquihue y Osorno no existe protección alguna para estos bosques (Wolordarsky-Franke y Lara, 2005).

Igualmente, pese a que los alerzales de la depresión intermedia cuentan con un $28,7 \%$ de protección, debido a su escasa superficie y nivel de fragmentación, resulta urgente otorgar una mayor protección a estos parches boscosos para evitar su desaparición.

En Chiloé y los Andes los alerzales se encuentran en teoría mejor protegidos (34,8\% y $48,6 \%$ de su superficie, respectivamente). En este contexto, es necesario destacar que, en el caso de la cordillera de los Andes, 44.496,2 ha de alerzales pasaron a manos del Estado chileno a través de la donación del Parque Pumalín durante el año 2019, lo que aumentó la superficie protegida de $22,3 \% 48,6 \%$ en este sector.

\section{Presión Sobre los Bosques de Alerce en la Región de Los Lagos}

Los bosques de alerce han sido históricamente explotados, especialmente desde los inicios de la república (Torrejón et al., 2011). Se estima que entre los años 1550 y 1997 los bosques de alerce disminuyeron en Chile de 617.077 a 264.993 ha (Lara et al., 1999). Dada la alta tasa de destrucción de estos bosques, la especie fue declarada como Monumento Natural a través del Decreto Supremo 490 en 1976, con lo cual se prohíbe su corta. Sin embargo, el artículo $5^{\circ}$ de dicho decreto permite la corta y utilización de madera "muerta" de alerce, es decir, se puede utilizar la madera de individuos que murieron antes de 1976, con lo que hasta la actualidad se ha estimulado la corta ilegal y los incendios intencionales para generar "madera muerta" que puede ser utilizada y vendida "legalmente" (Wolodarsky-Franke y Lara 2003, 2005).

Actualmente existe un total de 3,89 millones de $\mathrm{m}^{3}$ de madera muerta de alerce en Chile (Bahamondez, 2007). Desde el año 1991 a 2012 las exportaciones de productos basados en madera de alerce han ido a la baja, y desde el año 2013 no hay registro de exportaciones de productos de la especie (base de datos exportaciones forestales INFOR), lo cual refleja que al menos en el mercado formal, no existe casi comercialización de la especie al exterior. Sin embargo, la magnitud del mercado nacional es una incógnita.

Al analizar la matriz circundante de los bosques de alerce, se puede inferir que especialmente los bosques de la depresión intermedia se encuentran bajo una gran presión, con $66,1 \%$ de su matriz circundante cubierta de matorrales y praderas (lo que indica un persistente cambio de uso del suelo cercano a estos bosques). De hecho, se registró la pérdida de un $46 \%$ del hábitat potencial de la especie en aproximadamente una década (período 19992011), la cual estuvo asociada al incremento en el número de parches de bosque nativo (fragmentación) en la zona (Rodríguez-Echeverry et al., 2015). Esta zona está bajo la presión creciente de las ciudades de Puerto Varas y Puerto Montt (Fraver et al., 1999) y la expansión inmobiliaria, con abundantes loteos de parcelas, amenaza fuertemente la persistencia de la especie.

En el caso de los alerzales de Chiloé, la alta participación de matorrales y praderas en la matriz circundante $(32,3 \%)$, se puede deber a condiciones naturales de estos bosques costeros de montaña, en que alerce crece asociado a vegetación turbosa de "campañas" (Ramírez et al., 2014). En los casos donde la matriz circundante corresponde a bosques, los renovales participan de forma importante en la matriz de los bosques de Sarao $(30,2 \%)$, de Chiloé $(19,2 \%)$ y la depresión intermedia $(89,8 \%)$. Esto significa que son bosques de segundo 
crecimiento que se han generado probablemente debido a incendios o tala indiscriminada en cada zona (Lara et al., 1999; raver et al., 1999; Urrutia, 2002). De esta forma, solo los bosques de la cordillera de Los Andes se encontrarían bajo una aparente menor presión, ya que un $84,2 \%$ de su matriz circundante está compuesta por bosque, y sobre el $89 \%$ de ellos se encuentra en estado adulto o achaparrado (estructuras asociadas a buen estado de conservación). Esto se da principalmente por el difícil acceso a los bosques de alerce de esta cordillera, lo cual los ha resguardado mejor de cortas ilegales e incendios intencionales en los últimos años.

Una presión extra que está sufriendo el alerce actualmente es el cambio climático, el cual ha afectado negativamente su crecimiento en la cordillera de la Costa desde el año 1970 (Urrutia-Jalabert et al., 2015b) y en algunos sectores de los Andes desde 1980 (Camarero y Fajardo, 2017). En otros sectores de la cordillera de Los Andes se ha producido un aumento sostenido en el crecimiento durante el siglo XX y hasta el año 2010, lo cual ha sido asociado al aumento en la radiación y a la fertilización por $\mathrm{CO}_{2}$ (Urrutia-Jalabert et al., 2015b; Lara et al., 2020). Sin embargo, este patrón de aumento se evaluó hasta hace una década atrás y es necesario estudiar si el crecimiento durante los últimos años en estos mismos sitios no se ha visto afectado por el calentamiento y las menores precipitaciones. Lo anterior es importante, pues alerce responde en forma negativa especialmente al aumento de las temperaturas y éstas se han incrementado sostenidamente desde que hay registro en Chile (Lara y Villalba, 1993; Urrutia-Jalabert et al., 2015b; Lara et al., 2020).

Por otra parte, se ha reportado que el alerce puede llegar a ser muy resistente a las condiciones de sequía, pero esto no es igual en todos los sitios y para las distintas edades (Urrutia-Jalabert et al., 2018; ; Urrutia-Jalabert et al., 2020). Es así como los alerces jóvenes creciendo en sitios restrictivos de la cordillera de la Costa, son mucho más vulnerables que los adultos, lo cual constituye una alerta acerca del futuro de los bosques si las condiciones secas se vuelven extremas (Urrutia-Jalabert et al., 2018a). Es reconocido que el alerce es una especie milenaria y que como tal puede tener muchas estrategias para hacer frente a condiciones climáticas extremas, sin embargo, no hay que descuidar su estudio y monitoreo, para que no suceda lo que con Araucaria araucana y la mortalidad observada en los últimos años (Lara et al., 2019).

Por último, un factor no menos relevante, sobre todo considerando las poblaciones de la cordillera de la Costa y la depresión intermedia, es la presión de utilización que existe sobre sectores con musgo (Sphagnum spp.) en los cuales se desarrolla alerce. Se ha demostrado que esta especie, aunque puede limitar el crecimiento de plantas ya establecidas (Lara et al., 2008), ayuda a mantener un buen estatus hídrico en estas, por lo que su extracción puede afectar negativamente la sobrevivencia de la especie bajo las condiciones actuales más secas y calurosas (Urrutia-Jalabert et al., 2018a).

\section{Desafíos para la Conservación y Restauración de los Alerzales de la Región de Los Lagos}

Los bosques dominados por alerce en la región de Los Lagos presentan condiciones de estructura, composición y origen biogeográfico-genético que ameritan una atención sectorizada para su conservación (Allnutt et al., 1999). Debido al bajo número de rodales, baja superficie, alta fragmentación y una matriz circundante dominada por praderas y matorrales, se debiera fomentar y aumentar la protección de los alerzales de la depresión intermedia. Para esto resulta recomendable identificar con vuelos de dron los fragmentos y cada uno de los individuos de esta especie, de forma de mapearlos y así evitar incluso la extracción de individuos aislados. Además, es necesario ejecutar ampliamente actividades de restauración 
financiadas por el Estado y el sector privado, e implementar acciones a nivel de paisaje de forma de aumentar el tamaño de los fragmentos, mejorar la conectividad entre estos y aumentar la viabilidad de que sean habitados por alerce (Rodriguez-Echeverry et al., 2015). La restauración de la especie podría seguir diseños similares a los ya implementados para otras coníferas de lento crecimiento como P. uviferum (Bannister, 2015).

Por otra parte, el nulo nivel de protección, alto grado de alteración, la ocurrencia periódica de talas ilegales y una matriz circundante muy alterada en torno a los alerzales de la cordillera de la costa de las provincias de Osorno y Llanquihue, hacen que aún sea una deuda pendiente del Estado de Chile declarar parte de este territorio como área protegida. Además, la restauración de estos bosques tanto dentro de su superficie cómo en su matriz circundante, son medidas que debieran comenzar a ejecutarse. Para esto, el fomento de la abundante regeneración natural (Bahamondez, 2007) podría ser un buen punto de partida. En el caso de los alerzales de Chiloé, pese a que aproximadamente un tercio de ellos se encuentran bajo protección en el Parque Nacional Chiloé, existen sectores aledaños al parque que poseen poco resguardo (ej. Sector Abtao y Huentemó-Chanquín), por lo que el riesgo de incendios es alto, más aún bajo las condiciones climáticas actuales. En los alerzales de la isla sería recomendable apoyar actividades de restauración en estos bosques afectados históricamente por incendios (Urrutia, 2002), a través de técnicas de restauración activa, pasiva y mixta, basadas en el fomento y asistencia de la regeneración natural que según estudios anteriores es bastante abundante (Bahamondez, 2007).

Según la información disponible, los alerzales que se encuentran en mejor estado de conservación son los de la cordillera de los Andes, pues presentan casi $50 \%$ de representatividad en SNASPE, existe un alto porcentaje de bosques adultos, existe menor fragmentación y la matriz circundante indica menor presión. Sin embargo, es importante señalar que la tala ilegal y los incendios pueden llegar a convertirse en una gran amenaza a medida que se va mejorando el acceso a los bosques de esta zona. Para evitar esto es muy importante fortalecer la fiscalización y el monitoreo especialmente en zonas donde hay caminos y huellas antiguas que llegan a los alerces (ej.: Contao).

Por último, en las próximas décadas la región de los Lagos sufrirá grandes cambios en su clima. Modelos predictivos existentes muestran que a fines de este siglo la temperatura aumentará en $2-4^{\circ} \mathrm{C}$ y la precipitación estival bajará en un $30-50 \%$ a esta latitud $\left(41-43^{\circ} \mathrm{S}\right.$, Universidad de Chile, 2006). ¿Cuál va a ser el efecto de estos cambios en los bosques de alerce?. Estudios han avanzado en la estimación de la vulnerabilidad de alerce al cambio climático (Urrutia-Jalabert et al., 2015b; Urrutia-Jalabert et al., 2018a; Urrutia-Jalabert et al., 2020); sin embargo, aún faltan investigaciones que puedan indagar potenciales cambios en la estructura, dinámica, flujos de agua y carbono, y en la persistencia de estos bosques en el largo plazo. La tarea es vasta, pero el fin último de comprender y resguardar este patrimonio de la humanidad bien lo amerita.

\section{RECONOCIMIENTOS}

INFOR y los autores agradecen el financiamiento entregado por el convenio INFORMINAGRI. Este estudio es parte del trabajo realizado por el programa de investigación en Restauración de Ecosistemas Forestales Nativos de INFOR. Igualmente se agradece al Proyecto de Inserción en el sector productivo PAI-ANID 7818120003, al Proyecto Fondecyt 1171496 y al Proyecto ANID/FONDAP 15110009. 


\section{REFERENCIAS}

Allnutt, T.R.; Newton, A.C.; Lara, A.; Premoli, A.; Armesto, J.J.; Vergara, R, y Gardner, M, 1999. Genetic variation in Fitzroya cupressoides (alerce), a threatened South American conifer, Molecular Ecology, 8: 975987.

Bahamondez, C, 2007. Inventario de los bosques de alerce. Santiago, Chile: Instituto Forestal, p. 183.

Bannister, J.R.; Vidal, O.J.; Teneb, E, y Sandoval, V, 2012. Latitudinal patterns and regionalization of plant diversity along a 4270-km gradient in continental Chile, Austral Ecology, 37:500-509.

Bannister, J.R, 2015. Recuperar bosques no es solo plantar árboles: Lecciones aprendidas luego de 7 años restaurando bosques de Pilgerodendron uviferum (D. Don) Florin en Chiloé', Anales del Instituto de la Patagonia, 43: 35-51.

Bannister, J.R, y Donoso, P.J, 2013. Forest typification to characterize the structure and composition of oldgrowth evergreen forests on Chiloe Island, North Patagonia (Chile), Forests, 4: 1087-1105.

Camarero, J.J, y Fajardo, A, 2017. Poor acclimation to current drier climate of the long-lived tree species Fitzroya cupressoides in the temperate rainforest of southern Chile. Agricultural and Forest Meteorology 239(1): 141-150

CONAF, 2020. Catastro de los Recursos Vegetacionales Nativos de Chile. Cifras oficiales 2017. Disponible en: http://www.conaf.cl/nuestros-bosques/bosques-en-chile/catastro-vegetacional.

Di Castri, F. y Hajek, E., 1976. Bioclimatología de Chile. Santiago, Chile: Vicerrectoría Académica de la Universidad Católica de Chile.

Donoso, C.; Grez, R.; Sandoval, V., 1990. Caracterización del Tipo Forestal Alerce. Bosque 11: 21-34.

Donoso, C., 1993. Producción de semillas y hojarasca de las especies del Tipo Forestal Alerce (Fitzroya cupressoides) de la Cordillera de la Costa de Valdivia, Chile. Revista Chilena de Historia Natural 66: 53-64.

Fraver, S.; González M.E.; Silla, F; Lara, A, y Gardner, M., 1999. Composition and structure of remnant Fitzroya cupressoides forests of Southern Chile's Central Depression. Journal of the Torrey Botanical Society 126: 49-57.

Kitzberger, T.; Perez, A.; Iglesias, G.; Premoli, A. y Veblen, T., 2000. Distribución y estado de conservación del alerce (Fitzroya cupressoides (Mol.) Johnst.) en Argentina. Bosque, 21: 79-89.

Kreft, H. y Jetz, W., 2010. A framework for delineating biogeographical regions based on species distributions, Journal of Biogeography, 37: 2029-2053.

Jung, M., 2013. LecoS - A python plugin for automated landscape ecology analysis. Ecological Informatics, 31: 18-21.

Lara, A. y Villalba, R., 1993. A 3,620-year temperature reconstruction from Fitzroya cupressoides tree rings in southern South America. Science 260: 1104-1106.

Lara, A; Fraver, S; Aravena, J.C, y Wolodarsky-Franke, A., 1999. Fire and the dynamics of Fitzroya cupressoides (Alerce) forests of Chile's Cordillera Pelada. Ecoscience 6, 100-109

Lara A.; Aravena, J.C.; Wolodarskay, A.; Cortés, M. y Fraver, S., 2003. Fire regimes and forest dynamics in the lake district in south-central Chile. In: T.T. Veblen, W. Baker, G. Montenegro \& T.W.Swetnam. Fire and 
Climatic Change in the Temperate Ecosystems of the Western Americas. Springer-Verlag. 322-342.

Lara, A.; Echeverría, C.; Thiers, O.; Huss, E.; Escobar, B.; Tripp, K.; Zamorano, C. y Altamirano, A, 2008. Restauración ecológica de coníferas longevas: el caso del alerce (Fitzroya cupressoides) en el sur de Chile. Páginas 39-56 en M. González-Espinosa, J. M. Rey-Benayas y N. Ramírez-Marcial, editores. Restauración de bosques en América Latina. Fundación Internacional para la Restauración de Ecosistemas (FIRE) y Editorial Mundi-Prensa México, México.

Lara, A.; Urrutia-Jalabert, R.; Reyes, R., González, M.; Miranda, A.; Altamirano, A. y Zamorano, C., 2019. Bosques Nativos, en: Informe País Estado del Medio Ambiente en Chile 2018. Instituto de Asuntos Públicos. Centro de Análisis de Políticas Públicas. Santiago, Chile.

Lara, A.; Villalba, R.; Urrutia-Jalabert, R.; González-Reyes, A.; Aravena, J. C.; Luckman, B. H.; Cuq, E.; Rodríguez, C. y Wolodarski-Franke, A., 2020. A 5680-year tree-ring temperature record for southern South America. Quaternary Science Reviews 228:106087.

Ramírez, C; San Martin, C; Vidal, O; Pérez, Y, Valenzuela, J, Solís, J.L, y Toledo, G, 2014. Tundra Subantártica en la Isla Grande de Chiloé, Chile: Flora y vegetación turbosa de campañas, Anales del Instituto de la Patagonia, 42: 17-37.

Rodríguez-Echeverry, J.; Echeverría, C. y Nahuelhual, L., 2015. Impacts of anthropogenic land-use change on populations of the Endangered Patagonian cypress Fitzroya cupressoides in southern Chile: Implications for its conservation. Oryx, 49(3), 447-452.

SERNAGEOMIN, 2003. Mapa Geológico de Chile, versión digital. Servicio Nacional de Geología y Minería.

Silla, F.; Fraver, S.; Lara, A.; Allnutt, T.R.; y Newton, A., 2002. Regeneration and stand dynamics of Fitzroya cupressoides (Cupressaceae) forest of southern Chile's Central Depression. Forest Ecology and Management 165: 213-224.

Torrejón, G.F.; Cisternas, M.; Alvial, I. y Torres, L., 2011. Consecuencias de la tala maderera colonial en los bosques de alerce de Chiloé, sur de Chile (Siglos XVI-XIX)', Magallania, 39: 75-95.

UICN, 2020. The UICN Red List of Threatened Species $2020 . \quad$ In: https://www.iucnredlist.org/species/30926/2798574 _ 10 de septiembre, 2020.

Universidad de Chile, 2006. Estudio de la variabilidad climática en Chile para el Siglo XXI. Informe Final. Santiago, Chile: Departamento de Geofísica. Universidad de Chile.

Urrutia, R., 2002. Desarrollo de una cronología de anchos de anillos para alerce (Fitzroya cupressoides) y reconstrucción de la historia de incendios en el área de Abtao, Parque Nacional Chiloé, $X$ región. Tesis Ingeniería Forestal. Pontificia Universidad Católica de Chile.

Urrutia-Jalabert, R.; Malhi, Y. y Lara, A., 2015a. The oldest, slowest rainforests in the world? massive biomass and slow carbon dynamics of Fitzroya cupressoides temperate forests in southern Chile. PLoS One 10:137569.

Urrutia-Jalabert, R,; Malhi, Y.; Barichivich, J.; Lara, A.; Delgado-Huertas, A.; Rodríguez, C. y Cuq, E., 2015b. Increased water use efficiency but contrasting tree growth patterns in Fitzroya cupressoides forests of southern Chile during recent decades. J. Geophys. Res. Biogeosci. 120, 2505-2524.

Urrutia-Jalabert, R.; Peña, M.P.; Coopman, R.E.; Carvajal, D.E.; y Jiménez-Castillo, M. $2018 a$. Elucidating the hydraulic vulnerability of the longest-lived Southern Hemisphere conifer to aridification. Forest Ecol. Manag. 430, 472-484.

Urrutia-Jalabert, R.; González, M.E.; González-Reyes, A.; Lara, A. y Garreaud, R., 2018b. Climate variability and forest fires in central and south-central Chile. Ecosphere 9:e02171. 
Urrutia-Jalabert, R.; Lara, A.; Barichivich, J.; Vergara, N.; Rodriguez, C.G.; y Piper, F., 2020. Low growth sensitivity and fast replenishment of non-structural carbohydrates in a long-lived endangered conifer after drought. Frontiers in Plant Science 11:905.

Veblen, T.; Delmastro, J. y Schlatter, J.E., 1976. The conservation of Fitzroya cupressoides and its environment in southern Chile. Enviromental Conservation, 3: 291-301.

Villagrán, C.; León, A. y Roig, F.A., 2004. Paleodistribución del alerce y ciprés de las Guaitecas durante períodos interestadiales de la Glaciación Llanquihue: provincias de Llanquihue y Chiloé, Región de Los Lagos, Chile, Revista geológica de Chile, 31: 133-151.

Wolodarsky-Franke, A. y Lara, A., 2003. La dendrocronología como herramienta en la conservación de especies arbóreas en peligro en Chile. Gestión Ambiental 9, 15-23.

Wolodarsky-Franke, A. y Lara, A., 2005. The role of "forensic" dendrochronology in the conservation of alerce (Fitzroya cupressoides (Molina Johnston) forests in Chile. Dendrochronologia 22:235-240. 